\title{
Innate signaling by the C-type lectin DC-SIGN dictates immune responses
}

\author{
Jeroen den Dunnen · Sonja I. Gringhuis • \\ Teunis B. H. Geijtenbeek
}

Received: 5 August 2008 / Accepted: 13 October 2008 / Published online: 8 November 2008

(C) The Author(s) 2008. This article is published with open access at Springerlink.com

\begin{abstract}
Effective immune responses depend on the recognition of pathogens by dendritic cells (DCs) through pattern recognition receptors (PRRs). These receptors induce specific signaling pathways that lead to the induction of immune responses against the pathogens. It is becoming evident that C-type lectins are also important PRRs. In particular, the C-type lectin DC-SIGN has emerged as a key player in the induction of immune responses against numerous pathogens by modulating TLR-induced activation. Recent reports have begun to elucidate the molecular mechanisms underlying these immune responses. Upon pathogen binding, DC-SIGN induces an intracellular signaling pathway with a central role for the serine/threonine kinase Raf- 1 . For several pathogens that interact with DC-SIGN, including Mycobacterium tuberculosis and HIV-1, Raf-1 activation leads to acetylation of NF- $\kappa \mathrm{B}$ subunit $\mathrm{p} 65$, which induces specific gene transcription profiles. In addition, other DC-SIGN-ligands induce different signaling pathways downstream of Raf-1, indicating that DC-SIGN-signaling is tailored to the pathogen. In this review we will discuss in detail the current knowledge about DC-SIGN signaling and its implications on immunity.
\end{abstract}

Keywords C-type lectin · DC-SIGN · HIV-1 · Innate signaling $\cdot$ Mycobacteria $\cdot$ Raf- 1

This article is a symposium paper from the conference "Immunotherapy-From Basic Research to Clinical Applications", Symposium of the Collaborative Research Center (SFB) 685, held in Tübingen, Germany, 6-7 March 2008.

J. den Dunnen · S. I. Gringhuis · T. B. H. Geijtenbeek ( () Department of Molecular Cell Biology and Immunology, VU University Medical Center, P.O. Box 7057, 1007 MC Amsterdam, The Netherlands e-mail: t.geijtenbeek@vumc.nl

\section{Introduction}

Effective immune responses depend on the recognition of pathogens by dendritic cells (DCs) through pattern recognition receptors (PRRs) [23]. These PRRs recognize socalled pattern associated molecular patterns (PAMPs), which are conserved groups of molecules from pathogens that are essential for microbial survival, such as bacterial or fungal cell wall components and viral or bacterial nucleic acids [23]. DCs are equipped with several different classes of PPRs that recognize distinct PAMPs expressed by pathogens. These different classes of PRRs include the Toll-like receptors (TLRs), NOD-like family receptors and CARD helicases. Activation of these receptors induces receptor-specific intracellular signals that regulate the expression of response genes, such as those encoding co-stimulatory molecules and cytokines and chemokines.

Most pathogens express different PAMPs and trigger several classes of PRRs on a single cell simultaneously. As a result, the ultimate expression of response genes induced by a pathogen depends on the integration of these different signaling pathways. In fact, cross-talk between or even within groups of PRRs is crucial in balancing immune responses through collaborative induction of positive or negative feedback mechanisms [27].

In recent years C-type lectins have emerged as PRRs that play key roles in the induction of immune responses against numerous pathogens. The C-type lectins DC-specific ICAM-3 grabbing non-integrin (DC-SIGN) and DC-associated C-type lectin-1 (Dectin-1) both shape immune responses against various pathogens. The intracellular signaling pathways induced by these $\mathrm{C}$-type lectins modulate the responses of other PRRs such as TLRs, but also exert functions independent from other PRRs [45]. 
DC-SIGN interacts with numerous different pathogens including (myco)bacteria, fungi, and viruses. Although the interaction of DC-SIGN with pathogens significantly affects the induction of immune responses [44], the molecular mechanisms that underlie DC-SIGN functions have remained elusive for years. Recently, several studies have shed light on the intracellular signaling pathways through which DC-SIGN shapes immune responses [18, 21, 22]. In this review we will discuss DC-SIGN signaling, with a major role for the mechanism of cross-talk between DC-SIGN and TLRs.

\section{Immunomodulation by DC-SIGN}

DC-SIGN is a calcium-dependent carbohydrate-binding protein expressed by DCs [15] and macrophage subpopulations [17] with a wide range of immunological functions. DC-SIGN acts as an adhesion receptor that interacts with ICAM-2 on endothelial cells to induce tethering and transendothelial migration of DCs [12] and mediates clustering of DCs with naive T cells through binding of ICAM-3 [15]. In addition, DC-SIGN functions as a PRR that induces specific immune responses upon interaction with numerous pathogens [44]. DC-SIGN binds distinct carbohydrate structures such as mannose-containing glycoconjugates [30] and fucose-containing blood-group antigens [1]. This carbohydrate recognition pattern is the basis of its broad specificity for different pathogens and might also be responsible for its distinct signaling properties.

Mycobacteria target DC-SIGN to affect TLR4-mediated immune responses by impairing DC-maturation and enhancing IL-10 production [14]. Similarly, DC-SIGN binding to the Lewis antigens on LPS from Helicobacter pylori induces IL-10 production but inhibits Th1 polarization [3]. In contrast, LPS from Neisseria meningitidis mutants skews $\mathrm{T}$ cells toward a Th1 response by binding to DC-SIGN [41]. In addition, DC-SIGN binding by specific Lactobacilli species induces regulatory $\mathrm{T}$ cell differentiation [40]. As binding of DC-SIGN by different pathogens results in distinct immunological outcomes, these findings support an important role for DC-SIGN as an immunomodulator.

Several mechanisms have been suggested to account for the observed modulation of TLR activation by DC-SIGN. Co-localization of DC-SIGN with TLR4 could result in enhanced TLR4 signaling, as has been described previously for SIGNR1, a murine homolog of DC-SIGN [31]. In contrast, DC-SIGN has been hypothesized to induce intracellular signaling itself that converges with signaling pathways of other PRRs.

Recent studies on the interaction of mycobacteria with DCs have shed some light on the molecular mechanisms that underlie DC-SIGN-mediated immune responses.
Mycobacteria such as Mycobacterium tuberculosis interact with DCs through various receptors, including TLRs and C-type lectins. TLR-triggering by mycobacteria induces DC maturation and the induction of specific cytokines. However, in addition to TLRs, mycobacteria also interact with DCs by binding to DC-SIGN. One of the main ligands responsible for mycobacterial binding to DC-SIGN is ManLAM [14], a cell wall component abundantly expressed by pathogenic mycobacterial species such as M. tuberculosis. The interaction of ManLAM with DC-SIGN increases the production of the immunosuppressive cytokine IL-10 [14]. By screening with a large panel of inhibitors [18], it was found that upon binding of ManLAM, DC-SIGN triggers an intracellular signaling cascade independent from other PRRs. Pivotal to DC-SIGN-signaling is the activation of serine/threonine kinase Raf-1. Ligand binding leads to activation of Raf-1 and inhibition of Raf-1 completely blocks DC-SIGN-mediated immune responses to mycobacteria. In the next paragraphs, we will discuss DC-SIGN signaling in detail. For clarity, we will first focus on the upstream signal transduction pathways that lead to Raf-1 activation; subsequently, we will discuss DC-SIGN signaling downstream of Raf-1 and how this leads to modulation of immune responses.

\section{DC-SIGN signaling upstream of Raf-1}

How binding of ManLAM or other ligands to DC-SIGN leads to the initiation of intracellular signaling is still unclear. DC-SIGN displays several motifs in its cytoplasmic tail that theoretically could be involved in the induction of intracellular signaling pathways. Two motifs, a di-leucine-based motif and a tri-acidic (Glu-Glu-Glu) cluster, are known to be required for internalization [8]. In addition, DC-SIGN bears a YxxL motif in its cytoplasmic tail, which is known to be required for signaling of two other C-type lectins, Dectin-1 and CLEC-2 [9, 11, 35]. However, although it could play a role in the induction of other intracellular signals [21], the tyrosine motif of DC-SIGN is not essential for the activation of Raf-1 [18]. In addition, both the tyrosine and the di-leucine motif are required for the association of the cytoplasmic tail of DC-SIGN with leukocyte specific protein 1 (LSP1), an F-actin binding protein [39]. However, LSP1 has not been established to play a role in signaling, but has been shown to mediate DC-SIGNdependent transport of HIV-1 to proteasomes [39]. Clustering of DC-SIGN-molecules could also play an important role in the induction of intracellular signals. DC-SIGN forms tetramers [30], which might allow the binding of adaptor-molecules that can bridge the cytoplasmic tails of two or more DC-SIGN molecules, as has been hypothesized for Dectin-1 signaling [35]. Taken together, the 
mechanism of how ligand-binding leads to the induction of signal transduction is still highly speculative.

Currently, our knowledge of upstream DC-SIGN-signaling begins with the direct upstream mediators of Raf-1 (Fig. 1). A prerequisite for Raf-1 activation is its interaction with the active form of Ras, which induces a conformational change in Raf-1. Ras is a small G protein that can mediate signaling by binding and hydrolysing GTP; the GTP-bound form of Ras is active and capable of binding Raf-1, whereas the GDP-bound form is inactive [48]. Indeed, the binding of ManLAM induces the GTP-bound and active form of Ras through a yet to be identified Ras guanine exchange factor (GEF). Also, inhibition of Ras function prevents DC-SIGN-induced upregulation of cytokines [18]. However, interaction between Raf-1 and GTPRas alone is insufficient to activate the kinase activity of Raf- 1 and additional phosphorylation of Raf- 1 is required. Several phosphorylation sites on Raf-1 have been identified that are involved in its kinase activity [48]. Two of these phosphorylation sites are tyrosines 340/341 (Tyr340/341) and serine 338 (Ser338) [48]. Phosphorylation of Tyr340/ 341 on Raf-1 in response to DC-SIGN signaling is carried out by a member of the Src family of tyrosine kinases [18], most likely Lyn, Hck or Fgr, which are the most abundant Src kinases in DCs [28]. Raf-1 phosphorylation on Ser338 is induced by p21-activated kinases (Paks) [48]. Paks are known to be activated by either Rac1 or Cdc42, both small GTPases from the Rho GTPase family [48]. Indeed, inhibition of Rho GTPases with toxin B blocks DC-SIGNinduced phosphorylation of Raf-1 on Ser338 [18]. Interestingly, HIV-1 binding to DC-SIGN activates yet another Rho GTPase, RhoA, which is mediated via leukemia-associated Rho GEF (LARG) [21]. Since HIV-1-induced immune responses are dependent on Raf-1 [18], these data suggest that phosphorylation of Raf- 1 at residue Ser338 is mediated via LARG-RhoA-dependent activation of Pak.

Thus, DC-SIGN induces the activation of three routes that converge to activate Raf-1 (Fig. 1): activation of Ras leads to binding to Raf-1 and induces conformational changes that allow for subsequent phosphorylation of Raf-1 by Src and Pak kinases; Src kinases induce the phosphorylation of Raf-1 at residue Tyr340/341, whereas Rho GTPase-dependent activation of Pak kinases results in phosphorylation of Raf-1 at Ser338.

\section{DC-SIGN signaling downstream of Raf-1}

As described above, activation of Raf- 1 is essential for DCSIGN-mediated immune responses. However, signaling downstream of Raf-1 appears to be dependent on the specific DC-SIGN-ligand involved. For example, DC-SIGN activation by a specific antibody denoted MR-1 results in activation of extracellular signal-regulated kinase (ERK) [5]. Although mitogen-activated protein kinase kinase (MEK), which can activate ERK [25], is a well-characterized possible downstream effector of Raf-1, it is unclear whether MR-1 also activates Raf-1. In addition, some studies have shown that a C-type lectin interaction with HIV-1 [37] or a glycoprotein allergen [38] induces ERK activation, although direct involvement of DC-SIGN has not been demonstrated in these studies. In contrast, other reports have demonstrated that binding of pathogenic ligands to DC-SIGN does not lead to activation of ERK [18, 22]. Hence, ERK activation might be dependent on the ligand binding to DC-SIGN, although certain contaminants in the ligand preparations might also explain some of these differential effects. Nevertheless, for most pathogens including M. tuberculosis and HIV-1, interaction with DC-SIGN modulates immune responses by targeting nuclear factor$\kappa \mathrm{B}(\mathrm{NF}-\kappa \mathrm{B})$. An exception is DC-SIGN-ligand Salp15, which will be discussed separately in a paragraph below.

$\mathrm{NF}-\kappa \mathrm{B}$ activation is crucial for the induction of DCmediated immune responses [32]. The NF- $\kappa$ B family is composed of several subunits that can form homodimers and heterodimers. The most abundant and prototypical form of NF- $\kappa \mathrm{B}$ is a heterodimer between $\mathrm{p} 65$ and $\mathrm{p} 50$, of which p65 is the transcriptionally active component. In unstimulated cells, p65 is inactive and resides primarily in the cytosol in complex with any of the inhibitory $\mathrm{I} \kappa \mathrm{B}$ proteins. Upon stimulation, the $\mathrm{I} \kappa \mathrm{B}$ proteins are degraded and the p65-p50 dimers translocate into the nucleus where they bind to target DNA sequences to influence gene expression. Several classes of PRRs induce the activation of NF- $\kappa$ B after pathogen recognition. For example, TLR triggering leads to p65 activation via both MyD88- and TRIF-dependent pathways [42], which induces the transcription of numerous target genes including cytokines and chemokines.

The activity of p65 is regulated by several post-translational modifications such as phosphorylation and acetylation [20]. Strikingly, DC-SIGN-signaling controls p65 activity by phosphorylation of p65 at serine 276 (Ser276), which is completely dependent on Raf-1-activation [18]. Subsequently, phosphorylation of p65 at Ser276 leads to acetylation of p65. Acetylation of p65 can be mediated by two histone acetyltransferases (HATs), CREB binding protein (CBP) and p300, which in human DCs show high constitutive activity (unpublished data). Acetylation is known to have major consequences for p65 activity [20]. DCSIGN-mediated acetylation of p65 leads to both increased and prolonged transcription from the $I L 10$ promoter, resulting in a strongly increased production of IL-10 by DCs [18].

Crucial for the understanding of DC-SIGN-signaling and its effect on immune responses is that DC-SIGN cannot 
Fig. 1 DC-SIGN signaling by mycobacteria, viruses and fungi. Binding of several pathogens including $M$. tuberculosis, C. albicans and $H I V-1$ to DC-SIGN activates three routes that converge to activate Raf-1: activation of Ras leads to binding to Raf- 1 and induces conformational changes that allow for subsequent phosphorylation of Raf- 1 by Src and Pak kinases; Src kinases induce the phosphorylation of Raf- 1 at residue Tyr340/341, whereas Rho GTPase-dependent activation of Pak kinases results in phosphorylation of Raf-1 at Ser338. After translocation of NF- $\kappa$ B by TLR-stimulation, DC-SIGNinduced Raf-1 activation mediates the phosphorylation of NF- $\kappa$ B subunit $\mathrm{p} 65$ at Ser276, which in turn leads to p65-acetylation. Acetylation of $\mathrm{p} 65$ both prolongs and increases IL-10 transcription, resulting in increased IL-10 production

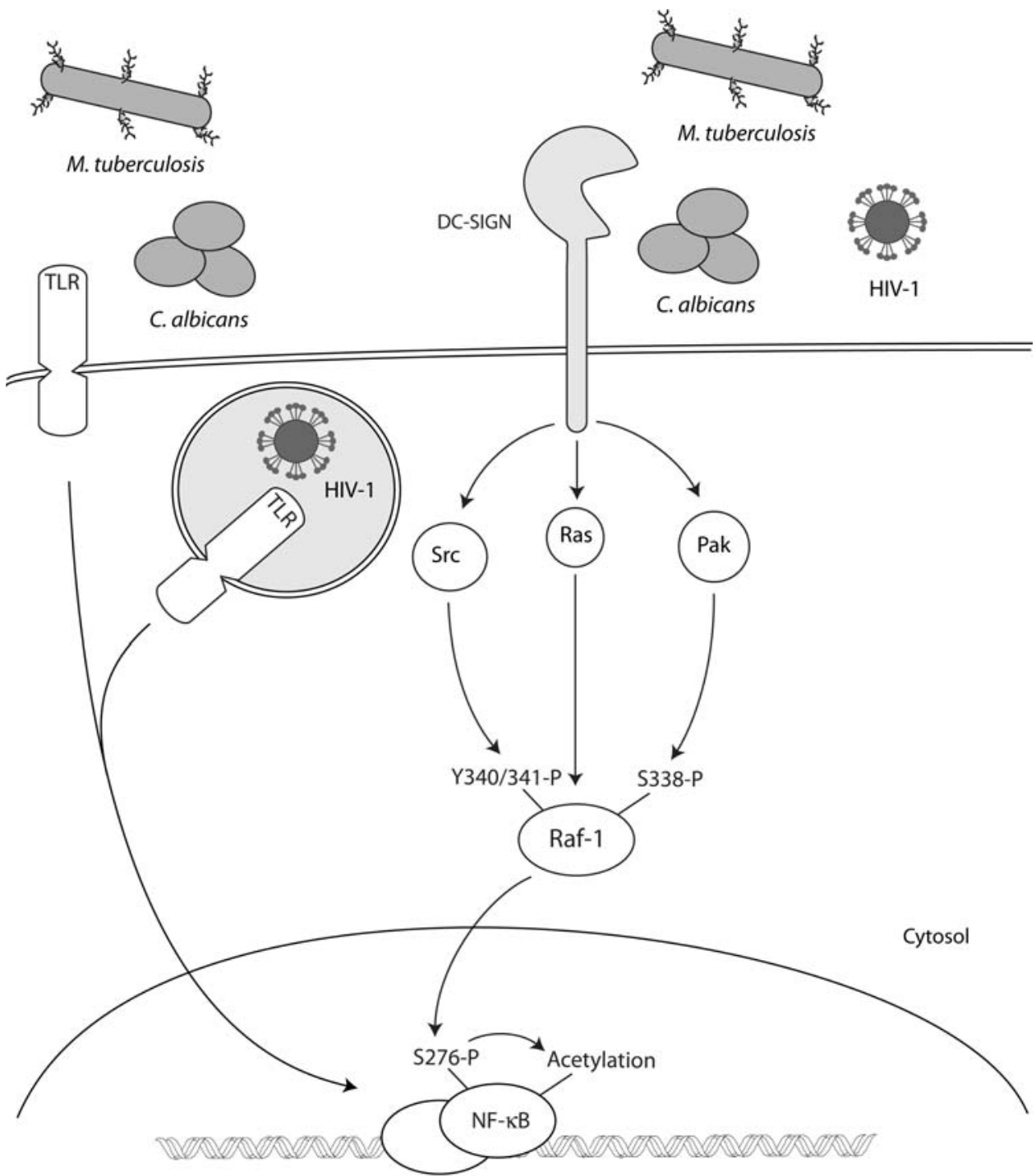

increased and prolonged IL-10 transcription Nucleus activate p65 by itself, but can only modulate p65-activity when activation of p65 has been induced by another receptor. The induction of DC-SIGN-signaling by the mycobacterial component ManLAM does not induce activation of p65, and therefore does not induce any cytokine responses. However, upon stimulation with whole mycobacteria, TLR4 and TLR2 triggering induce the activation and translocation of p65 to the nucleus, which allows for subsequent phosphorylation and acetylation of p65 by DC-SIGN signaling (Fig. 1).

A question that remains unanswered is the identity of the kinase responsible for phosphorylation of Ser276 of p65 upon DC-SIGN triggering. A number of kinases have previously been reported to phosphorylate Ser276 on p65, including protein kinase A (PKA) and the related mitogenand stress-activated kinase-1 (MSK1) [47, 49], but inhibi- tors against these kinases do not inhibit ManLAM-induced IL-10 upregulation [18]. Hence, the identity of this kinase is still under investigation.

\section{DC-SIGN-mediated modulation of immune responses to pathogens}

In addition to several mycobacterial species including M. tuberculosis, M. bovis BCG and M. leprae, DC-SIGNsignaling is induced by several other classes of pathogens. Yeasts (Candida albicans and Saccharomyces cerevisiae) and viruses (HIV-1 and measles virus) also induce the Raf1 pathway through DC-SIGN to modulate TLR responses [18]. This underscores that DC-SIGN has an important role in the regulation of DC-mediated immune responses to 
numerous pathogens. As mentioned before, immune modulation of NF- $\kappa$ B activation only occurs when a pathogen triggers DC-SIGN simultaneously with another receptor that activates NF- $\kappa$ B. For example, mycobacteria such as M. tuberculosis and $M$. bovis BCG activate NF- $\kappa \mathrm{B}$ through TLR2 and TLR4 activation, while viruses activate NF- $\kappa \mathrm{B}$ via TLR3 and TLR7/8 triggering in endosomes [6]. However, the modulation of immune responses by DC-SIGN is probably not restricted to TLR-activation, but instead might also include other NF- $\kappa \mathrm{B}$ dependent responses. Hypothetically, DC-SIGN signaling modulates the induction of any immune response that is p65-dependent, since phosphorylation and acetylation of p65 drastically alter the transcription rate, the duration of $\mathrm{p} 65$ activation and even the affinity of p65 with other proteins [20].

Additional investigations are required to determine how DC-SIGN signaling affects the immune response against a specific pathogen in toto. Besides the immunosuppressive cytokine IL-10, acetylation of p65 most likely modulates the transcription of other, pro-inflammatory immune genes as well, since the majority of cytokines induced by TLRstimulation in DCs depends at some level on NF- $\kappa \mathrm{B}$ activation. Therefore, the resulting net immune response induced by DC-SIGN-signaling depends on the complete array of cytokines that are modulated by p65-acetylation. Future investigations will be required to determine whether DC-SIGN-signaling dictates pro- or anti-inflammatory responses or whether it directs a specific type of $\mathrm{T}$ cell differentiation such as $\mathrm{T}$ helper $1,2,17$ or regulatory $\mathrm{T}$ cells.

\section{Immunomodulation by salivary tick protein Salp15}

While interaction with the previously described mycobacteria, viruses and fungi with DC-SIGN leads to Raf-1-dependent
Fig. 2 DC-SIGN signaling by Salp15. Salp15 from tick saliva modulates immune responses to tick-transmitted pathogens such as B. burgdorferi. Salp15 binding to DC-SIGN activates Raf-1 kinase similar as described for pathogens. However, in contrast to DC-SIGN-induced Raf-1 activation by pathogens, Salp15induced Raf- 1 activation leads to activation of MEK.

Salp15-induced Raf-1/MEK signaling regulates cytokine expression at different levels: inhibition of IL- 6 and TNF $\alpha$ is caused by enhanced degradation of their respective mRNAs, while decreased production of IL-12 results from impaired nucleosome remodeling at the IL-12p35 promoter

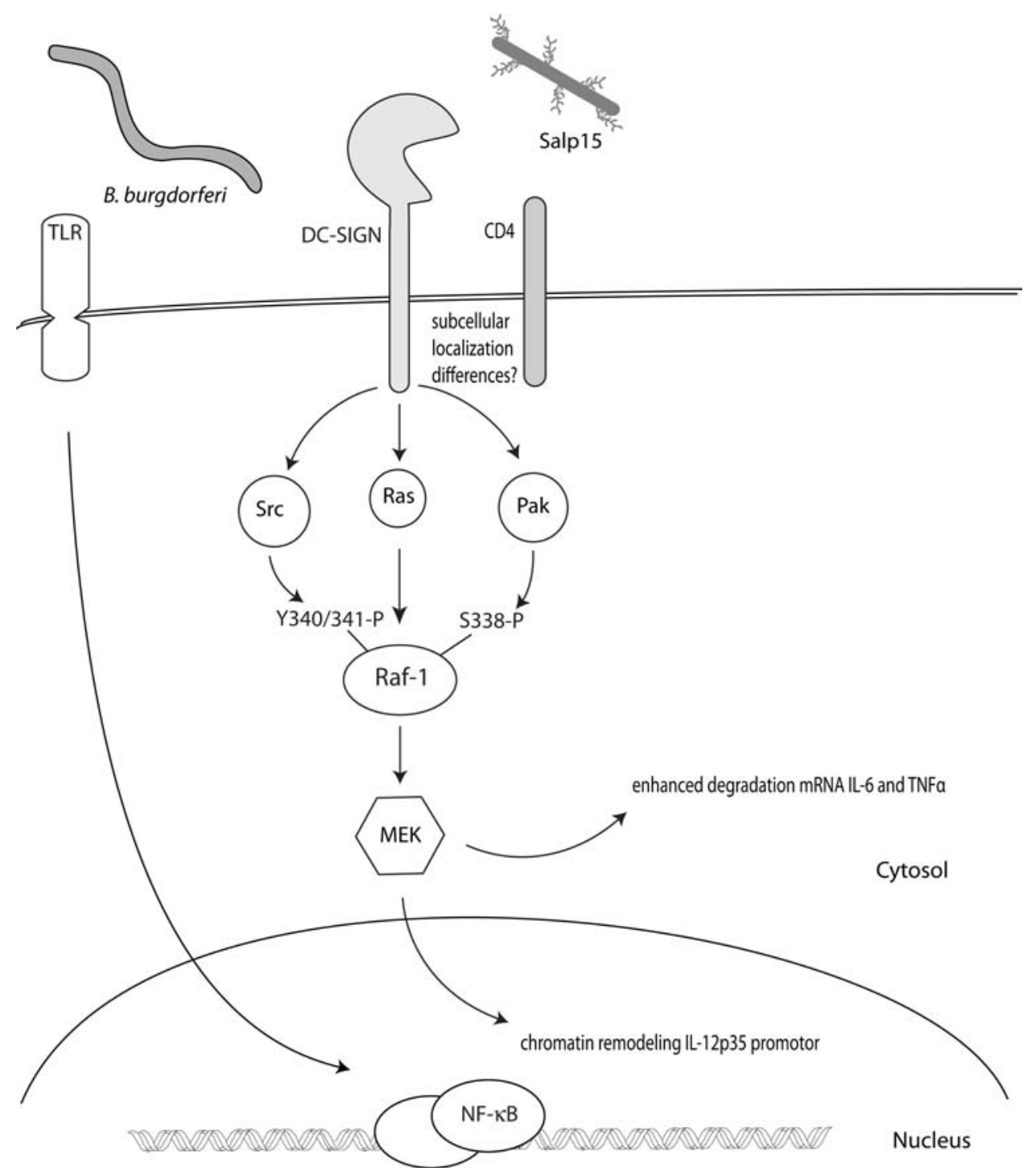


acetylation of NF- $\kappa \mathrm{B}$, interaction of another DC-SIGNligand, Salp15, modulates TLR-activation on DCs through a different mechanism. Salp15 is an immunomodulatory protein produced by the salivary glands of Ixodes scapularis ticks [7]. The presence of immunosuppressive molecules in tick saliva facilitate tick feeding, but also greatly enhance the tick's ability to transmit human pathogens such as Borrelia burgdorferi [33], the causative agent of Lyme disease.

Salp15 inhibits TLR2- and TLR4-induced production of pro-inflammatory cytokines IL-12, IL-6 and TNF $\alpha$. Similar to ManLAM, modulation of TLR-responses is completely dependent on the activation of Raf-1 [22]. However, in contrast to ManLAM, Salp15-induced signaling does not lead to phosphorylation or acetylation of p65, but instead to activation of MEK. The Salp15-induced Raf-1/MEK signaling regulates the inhibition of pro-inflammatory cytokines at different levels: inhibition of IL- 6 and TNF $\alpha$ is caused by enhanced degradation of their respective mRNAs, while decreased production of IL-12 results from impaired nucleosome remodeling at the $I L-12 p 35$ promoter (Fig. 2) [22].

The reason behind the different downstream effectors of Raf-1 after activation by Salp15 compared to ManLAM is still unclear. One explanation could be that Salp15 also activates other receptors, which modulate DC-SIGN signaling. Besides DC-SIGN, Salp15 has also been described to bind to CD4 [24]. Although no direct physical association has been found between both proteins [4], DC-SIGN and CD4 have been found to colocalize on the cell-surface [26]. On T cells, binding of Salp15 to CD4 modulates actin polymerization [24]. A central theme in signaling is bringing together kinases and substrates, and impairment of actin polymerization might bring Raf-1 together with a different downstream effector compared to ManLAM-induced DCSIGN-signaling. Therefore, co-ligation of DC-SIGN and CD4 by Salp15 might be responsible for the induction of this distinct signaling pathway by affecting the subcellular localization of either Raf-1 or its downstream effectors. Additionally, future research on the molecular mechanism of Salp15-induced DC immunosuppression is required to elucidate how MEK affects mRNA stability and nucleosome remodeling. Although MEK kinases are well known to activate ERK 1 and 2, Salp15 does not signal through ERK kinases [22]. The downstream effectors of MEK are most likely involved in post-translational modifications of the proteins involved in mRNA decay and nucleosome remodeling.

Thus, although Salp15 binding to DC-SIGN does induce Raf-1 activation similar to ManLAM, the downstream effectors that modulate TLR-induced activation are different. This demonstrates that pathogen binding to DC-SIGN might lead to different ligand-specific signaling cascades that regulate distinct adaptive immune responses. Although
Raf-1 seems to play a central role in DC-SIGN-induced modulation of TLR-responses, the downstream effectors of Raf-1 regulate the subsequent cytokine responses.

\section{Immune suppression and viral transmission by HIV-1}

As described previously, binding of HIV-1 to DC-SIGN modulates TLR activation via Raf-1 signaling [18]. However, DC-SIGN signaling by HIV-1 not only enhances TLR-induced IL-10 production, but also impairs TLRinduced dendrite formation of DCs and T cell proliferation [21]. In addition, HIV-1-induced DC-SIGN signaling mediates effects independent of TLR activation. Microarray studies revealed that cross-linking of DC-SIGN with an antibody that mimics HIV-1-binding specifically affected the gene expression profile of DCs, with hundreds of genes being induced or downregulated after ligation [21]. One of the most notable features of the DC-SIGN signaling program is the induction of ATF3, a negative regulator of TLR4 [16], suggesting that DC-SIGN signaling besides modulating also represses TLR4 signaling.

Besides modulation of immune responses, DC-SIGN signaling also plays an important role in the transmission of viruses from DCs to T cells [13]. DCs capture HIV-1 through the high-affinity interaction of DC-SIGN with gp120, the HIV-1 envelope glycoprotein. Subsequently, DCs are able to mediate transmission of the virus to T cells through the formation of a so-called infectious synapse between HIV-1-infected DCs and CD4+ T cells [29]. Recently, Hodges et al. [21] demonstrated that DC-SIGNinduced signaling is responsible for viral synapse formation between DCs and T cells. The authors found that binding of HIV-1 to DC-SIGN on DCs induces LARG-mediated activation of the GTPase RhoA, which is required for viral synapse formation. It will be interesting to investigate whether RhoA activation by HIV-1 is also involved in the Raf-1dependent immune responses (Fig. 1). Taken together, these findings suggest that the DC-SIGN signaling pathway orchestrates several immunological functions of DCs ranging from modulation of immune responses to the transmission of viruses to T cells.

\section{DC-SIGN signaling in context of other C-type lectins}

The C-type lectin receptor family has been increasingly appreciated as an important member of PRRs. However, although most $\mathrm{C}$-type lectins express several potential signaling motifs in their cytoplasmic domains, the molecular mechanisms underlying the C-type lectin functions are still largely unknown. The current knowledge about the molecular mechanisms of C-type lectins seems to suggest that the 
different receptors induce distinct signaling pathways. Recently, several studies have characterized the signaling pathways of Dectin-1, a C-type lectin involved in innate immune responses to fungal pathogens. Engagement of Dectin- 1 by fungal $\beta$-glucans leads to activation of spleen tyrosine kinase (Syk) [35], which is also induced upon ligation of CLEC-2 [9], but not DC-SIGN [9, 18]. Subsequently, Syk activation by Dectin-1 induces caspase recruitment domain 9 (Card9)-dependent activation of NF$\kappa \mathrm{B}$ [19] (for an extensive review on the mechanism of Dectin-1 signaling see [34]). As a consequence the activation of Dectin-1, in contrast to DC-SIGN signaling, directly leads to the expression of cytokines independent from TLR activation.

However, DC-SIGN and Dectin-1 also mediate similar functions. Analogous to DC-SIGN, Dectin-1 collaborates with TLRs for the induction of cytokines [10]. The signaling pathway underlying this cross-talk between Dectin-1 and TLRs remains to be identified, but seems to be independent of Syk [10, 35]. Since DC-SIGN and Dectin-1 bear common motifs in their cytoplasmic tail, this could suggest that Dectin-1 might use similar signaling pathways for the modulation of TLR responses. In addition, a recent study reported that both Dectin-1 and DC-SIGN mediate the induction of arachidonic acid [43]. Moreover, Dectin-1 was found to associate with DC-SIGN, which was enhanced upon stimulation with zymosan, a ligand for both Dectin-1 and DC-SIGN [43]. Since DC-SIGN and Dectin-1 are both involved in the recognition of pathogens such as C. albicans, co-localization of these two C-type lectins might add additional variability to both DC-SIGN- and Dectin-1induced signaling cascades.

\section{DC-SIGN signaling: beneficial for host or pathogen?}

DC-SIGN-signaling by pathogens does not modulate immune responses in the same manner, but instead the immune modulation depends on the pathogen involved. These differences most likely result from the specific set of PRRs that is activated. Every type of pathogen triggers a specific set of PRRs, which are modulated differently by DC-SIGN signaling. In addition, DC-SIGN itself might induce different signaling pathways depending on the DCSIGN ligand involved. However, a pivotal question that remains is whether the DC-SIGN-induced modulation of immune responses is beneficial for the host or whether DCSIGN signaling is induced by pathogens to subvert an effective immune response and promote their growth and survival.

In the case of HIV-1, DC-SIGN signaling appears to be unambiguously beneficial for the pathogen. DC-SIGN signaling by HIV-1 impairs DC maturation, T cell prolifer- ation and mediates the transmission of HIV-1 to T cells, thereby promoting systemic infection of the host. In addition, for mycobacteria DC-SIGN signaling was initially thought to be a mechanism through which DC function was suppressed to promote pathogen survival, based on impaired DC maturation and enhanced production of the immunosuppressive cytokine IL-10 by DCs after stimulation with ManLAM. However, this suppression might also be beneficial to the host by preventing excessive immune activation. So far, genetic epidemiology has yielded conflicting data. A recent study suggested that a single nucleotide polymorphism (SNP) that leads to decreased DC-SIGN expression is associated with reduced risk for cavitary tuberculosis disease [46]; in contrast, SNPs in the DCSIGN promoter region that lead to increased DC-SIGN expression appear to be protective against tuberculosis [2]. In addition, in a recent study it was reported that transgenic mice expressing human DC-SIGN actually show enhanced clearance of mycobacteria [36]. DCs from DC-SIGN-mice displayed reduced tissue damage and prolonged survival. These findings suggest that instead of favoring the immune evasion of bacteria, DC-SIGN signaling may promote protection by limiting tuberculosis-induced pathology. However, remarkably, most DC-SIGN-binding pathogens are microbes that in general induce latent infections, such as M. tuberculosis, M. leprae, C. albicans and H. pylori. This suggests that suppression of the immune response via interaction with DC-SIGN results in a "steady-state" that attenuates immune-activation in order to limit detrimental side effects of a full-blown immune response, but in turn also allows for pathogen survival.

Acknowledgments We would like to thank M. A. W. P. de Jong and M. van der Vlist for critically reading the manuscript and helping with the illustrations. J. d. D. is supported by grants from the Dutch Scientific Research program (NWO 912-04-025). SIG is supported by the Dutch Asthma Foundation (3.2.03.39).

Open Access This article is distributed under the terms of the Creative Commons Attribution Noncommercial License which permits any noncommercial use, distribution, and reproduction in any medium, provided the original author(s) and source are credited.

\section{References}

1. Appelmelk BJ, Van Die I, van Vliet SJ, Vandenbroucke-Grauls CM, Geijtenbeek TB, van Kooyk Y (2003) Cutting edge: carbohydrate profiling identifies new pathogens that interact with dendritic cell-specific ICAM-3-grabbing nonintegrin on dendritic cells. J Immunol 170:1635

2. Barreiro LB, Neyrolles O, Babb CL, Tailleux L, Quach H, McElreavey K, Helden PD, Hoal EG, Gicquel B, Quintana-Murci L (2006) Promoter variation in the DC-SIGN-encoding gene CD209 is associated with tuberculosis. PLoS Med 3:e20

3. Bergman MP, Engering A, Smits HH, van Vliet SJ, van Bodegraven AA, Wirth HP, Kapsenberg ML, Vandenbroucke-Grauls CM, 
van Kooyk Y, Appelmelk BJ (2004) Helicobacter pylori modulates the $\mathrm{T}$ helper cell $1 / \mathrm{T}$ helper cell 2 balance through phasevariable interaction between lipopolysaccharide and DC-SIGN. J Exp Med 200:979

4. Bernhard OK, Lai J, Wilkinson J, Sheil MM, Cunningham AL (2004) Proteomic analysis of DC-SIGN on dendritic cells detects tetramers required for ligand binding but no association with CD4. J Biol Chem 279:51828

5. Caparros E, Munoz P, Sierra-Filardi E, Serrano-Gomez D, Puig-Kroger A, Rodriguez-Fernandez JL, Mellado M, Sancho J, Zubiaur M, Corbi AL (2006) DC-SIGN ligation on dendritic cells results in ERK and PI3K activation and modulates cytokine production. Blood 107:3950

6. Cunningham AL, Harman AN, Donaghy H (2007) DC-SIGN 'AIDS' HIV immune evasion and infection. Nat Immunol 8:556

7. Das S, Banerjee G, DePonte K, Marcantonio N, Kantor FS, Fikrig E (2001) Salp25D, an Ixodes scapularis antioxidant, is 1 of 14 immunodominant antigens in engorged tick salivary glands. J Infect Dis 184:1056

8. Engering A, Geijtenbeek TB, van Vliet SJ, Wijers M, van Liempt E, Demaurex N, Lanzavecchia A, Fransen J, Figdor CG, Piguet V, van Kooyk Y (2002) The dendritic cell-specific adhesion receptor DC-SIGN internalizes antigen for presentation to T cells. J Immunol 168:2118

9. Fuller GL, Williams JA, Tomlinson MG, Eble JA, Hanna SL, Pohlmann S, Suzuki-Inoue K, Ozaki Y, Watson SP, Pearce AC (2007) The C-type lectin receptors CLEC-2 and Dectin-1, but not DC-SIGN, signal via a novel YXXL-dependent signaling cascade. J Biol Chem 282:12397

10. Gantner BN, Simmons RM, Canavera SJ, Akira S, Underhill DM (2003) Collaborative induction of inflammatory responses by dectin-1 and Toll-like receptor 2. J Exp Med 197:1107

11. Gantner BN, Simmons RM, Underhill DM (2005) Dectin-1 mediates macrophage recognition of Candida albicans yeast but not filaments. EMBO J 24:1277

12. Geijtenbeek TB, Krooshoop DJ, Bleijs DA, van Vliet SJ, van Duijnhoven GC, Grabovsky V, Alon R, Figdor CG, van Kooyk Y (2000) DC-SIGN-ICAM-2 interaction mediates dendritic cell trafficking. Nat Immunol 1:353

13. Geijtenbeek TB, Kwon DS, Torensma R, van Vliet SJ, van Duijnhoven GC, Middel J, Cornelissen IL, Nottet HS, KewalRamani VN, Littman DR, Figdor CG, van Kooyk Y (2000) DC-SIGN, a dendritic cell-specific HIV-1-binding protein that enhances transinfection of T cells. Cell 100:587

14. Geijtenbeek TB, van Vliet SJ, Koppel EA, Sanchez-Hernandez M, Vandenbroucke-Grauls CM, Appelmelk B, van Kooyk Y (2003) Mycobacteria target DC-SIGN to suppress dendritic cell function. J Exp Med 197:7

15. Geijtenbeek TBH, Torensma R, van Vliet SJ, van Duijnhoven GCF, Adema GJ, van Kooyk Y, Figdor CG (2000) Identification of DC-SIGN, a novel dendritic cell-specific ICAM-3 receptor that supports primary immune responses. Cell 100:575

16. Gilchrist M, Thorsson V, Li B, Rust AG, Korb M, Roach JC, Kennedy K, Hai T, Bolouri H, Aderem A (2006) Systems biology approaches identify ATF3 as a negative regulator of Toll-like receptor 4 . Nature 441:173

17. Granelli-Piperno A, Pritsker A, Pack M, Shimeliovich I, Arrighi JF, Park CG, Trumpfheller C, Piguet V, Moran TM, Steinman RM (2005) Dendritic cell-specific intercellular adhesion molecule 3grabbing nonintegrin/CD209 is abundant on macrophages in the normal human lymph node and is not required for dendritic cell stimulation of the mixed leukocyte reaction. J Immunol 175:4265

18. Gringhuis SI, den Dunnen J, Litjens M, van het Hof B, van Kooyk Y, Geijtenbeek TBH (2007) C-type lectin DC-SIGN modulates toll-like receptor signaling via Raf-1 kinase-dependent acetylation of transcription factor NF-kappa B. Immunity 26:605
19. Gross O, Gewies A, Finger K, Schafer M, Sparwasser T, Peschel C, Forster I, Ruland J (2006) Card9 controls a non-TLR signalling pathway for innate anti-fungal immunity. Nature 442:651

20. Hayden MS, Ghosh S (2004) Signaling to NF-kappaB. Genes Dev 18:2195

21. Hodges A, Sharrocks K, Edelmann M, Baban D, Moris A, Schwartz O, Drakesmith H, Davies K, Kessler B, McMichael A, Simmons A (2007) Activation of the lectin DC-SIGN induces an immature dendritic cell phenotype triggering Rho-GTPase activity required for HIV-1 replication. Nat Immunol 8:569

22. Hovius JW, de Jong MA, den Dunnen J, Litjens M, Fikrig E, van der Poll T, Gringhuis SI, Geijtenbeek TB (2008) Salp15 binding to DC-SIGN inhibits cytokine expression by impairing both nucleosome remodeling and mRNA stabilization. PLoS Pathog 4:e31

23. Janeway CA, Medzhitov R (2002) Innate immune recognition. Annu Rev Immunol 20:197

24. Juncadella IJ, Garg R, Ananthnarayanan SK, Yengo CM, Anguita J (2007) T-cell signaling pathways inhibited by the tick saliva immunosuppressor, Salp15. FEMS Immunol Med Microbiol 49:433

25. Kolch W (2005) Coordinating ERK/MAPK signalling through scaffolds and inhibitors. Nat Rev Mol Cell Biol 6:827

26. Lee B, Leslie G, Soilleux E, O’Doherty U, Baik S, Levroney E, Flummerfelt K, Swiggard W, Coleman N, Malim M, Doms RW (2001) cis Expression of DC-SIGN allows for more efficient entry of human and simian immunodeficiency viruses via CD4 and a coreceptor. J Virol 75:12028

27. Lee MS, Kim YJ (2007) Signaling pathways downstream of pattern-recognition receptors and their cross talk. Annu Rev Biochem 76:447

28. Lowell CA (2004) Src-family kinases: rheostats of immune cell signaling. Mol Immunol 41:631

29. McDonald D, Wu L, Bohks SM, KewalRamani VN, Unutmaz D, Hope TJ (2003) Recruitment of HIV and its receptors to dendritic cell-T cell junctions. Science 300:1295

30. Mitchell DA, Fadden AJ, Drickamer K (2001) A novel mechanism of carbohydrate recognition by the C-type lectins DC-SIGN and DC-SIGNR. Subunit organization and binding to multivalent ligands. J Biol Chem 276:28939

31. Nagaoka K, Takahara K, Tanaka K, Yoshida H, Steinman RM, Saitoh S, kashi-Takamura S, Miyake K, Kang YS, Park CG, Inaba K (2005) Association of SIGNR1 with TLR4-MD-2 enhances signal transduction by recognition of LPS in gram-negative bacteria. Int Immunol 17:827

32. Ouaaz F, Arron J, Zheng Y, Choi Y, Beg AA (2002) Dendritic cell development and survival require distinct NF-kappaB subunits. Immunity 16:257

33. Ramamoorthi N, Narasimhan S, Pal U, Bao F, Yang XF, Fish D, Anguita J, Norgard MV, Kantor FS, Anderson JF, Koski RA, Fikrig E (2005) The Lyme disease agent exploits a tick protein to infect the mammalian host. Nature 436:573

34. Robinson MJ, Sancho D, Slack EC, LeibundGut-Landmann S, Sousa CRE (2006) Myeloid C-type lectins in innate immunity. Nat Immunol 7:1258

35. Rogers NC, Slack EC, Edwards AD, Nolte MA, Schulz O, Schweighoffer E, Williams DL, Gordon S, Tybulewicz VL, Brown GD, Sousa ReisE (2005) Syk-dependent cytokine induction by Dectin-1 reveals a novel pattern recognition pathway for $\mathrm{C}$ type lectins. Immunity 22:507

36. Schaefer M, Reiling N, Fessler C, Stephani J, Taniuchi I, Hatam F, Yildirim AO, Fehrenbach H, Walter K, Ruland J, Wagner H, Ehlers S, Sparwasser T (2008) Decreased pathology and prolonged survival of human DC-SIGN transgenic mice during mycobacterial infection. J Immunol 180:6836

37. Shan M, Klasse PJ, Banerjee K, Dey AK, Iyer SP, Dionisio R, Charles D, Campbell-Gardener L, Olson WC, Sanders RW, Moore 
JP (2007) HIV-1 gp120 mannoses induce immunosuppressive responses from dendritic cells. PLoS Pathog 3:e169

38. Shreffler WG, Castro RR, Kucuk ZY, Charlop-Powers Z, Grishina G, Yoo S, Burks AW, Sampson HA (2006) The major glycoprotein allergen from Arachis hypogaea, Ara h 1, is a ligand of dendritic cell-specific ICAM-grabbing nonintegrin and acts as a Th2 adjuvant in vitro. J Immunol 177:3677

39. Smith AL, Ganesh L, Leung K, Jongstra-Bilen J, Jongstra J, Nabel GJ (2007) Leukocyte-specific protein 1 interacts with DC-SIGN and mediates transport of HIV to the proteasome in dendritic cells. J Exp Med 204:421

40. Smits HH, Engering A, van der Kleij D, de Jong EC, Schipper K, van Capel TM, Zaat BA, Yazdanbakhsh M, Wierenga EA, van Kooyk Y, Kapsenberg ML (2005) Selective probiotic bacteria induce IL-10-producing regulatory $\mathrm{T}$ cells in vitro by modulating dendritic cell function through dendritic cell-specific intercellular adhesion molecule 3-grabbing nonintegrin. J Allergy Clin Immunol 115:1260

41. Steeghs L, van Vliet SJ, Uronen-Hansson H, van Mourik A, Engering A, Sanchez-Hernandez M, Klein N, Callard R, van Putten JP, van der LP, van Kooyk Y, van de Winkel JG (2006) Neisseria meningitidis expressing lgtB lipopolysaccharide targets DC-SIGN and modulates dendritic cell function. Cell Microbiol 8:316

42. Takeda K, Akira S (2004) TLR signaling pathways. Semin Immunol $16: 3$
43. Valera I, Fernandez N, Trinidad AG, Alonso S, Brown GD, Alonso A, Crespo MS (2008) Costimulation of dectin-1 and DC-SIGN triggers the arachidonic acid cascade in human monocytederived dendritic cells. J Immunol 180:5727

44. van Kooyk Y, Geijtenbeek TB (2003) DC-SIGN: escape mechanism for pathogens. Nat Rev Immunol 3:697

45. van Vliet SJ, den Dunnen J, Gringhuis SI, Geijtenbeek TB, van Kooyk Y (2007) Innate signaling and regulation of dendritic cell immunity. Curr Opin Immunol 19:435

46. Vannberg FO, Chapman SJ, Khor CC, Tosh K, Floyd S, JacksonSillah D, Crampin A, Sichali L, Bah B, Gustafson P, Aaby P, McAdam KP, Bah-Sow O, Lienhardt C, Sirugo G, Fine P, Hill AV (2008) CD209 genetic polymorphism and tuberculosis disease. PLOS ONE 3:e1388

47. Vermeulen L, de Wilde G, van Damme P, Vanden Berghe W, Haegeman G (2003) Transcriptional activation of the NF-kappaB p65 subunit by mitogen- and stress-activated protein kinase-1 (MSK1). EMBO J 22:1313

48. Wellbrock C, Karasarides M, Marais R (2004) The RAF proteins take centre stage. Nat Rev Mol Cell Biol 5:875

49. Zhong HH, May MJ, Jimi E, Ghosh S (2002) The phosphorylation status of nuclear NF-kappa B determines its association with CBP/ p300 or HDAC-1. Mol Cell 9:625 